\title{
Magnetic and transport properties controlled by structural disorder in $\mathrm{La}_{0.7} \mathrm{Ca}_{0.3} \mathrm{MnO}_{3}$ films
}

\author{
V.G. Prokhorov, V.A. Komashko, and G.G. Kaminsky \\ Institute of Metal Physics, National Academy of Sciences of Ukraine, Kiev 03142, Ukraine \\ E-mail: pvg@imp.kiev.ua
}

V.L. Svetchnikov

NCHREM, Rotterdamseweg 137, 2628AL, Delft, The Netherlands

Y.P. Lee and S.Y. Park

Quantum Photonic Science Research Center and Department of Physics, Hanyang University Seoul 133-791, Korea

Received December 19, 2003, revised April 1, 2004

\begin{abstract}
The magnetic properties of an amorphous, a partially-disordered, and a lattice-strained crystalline $\mathrm{La}_{0.7} \mathrm{Ca}_{0.3} \mathrm{MnO}_{3}$ film are investigated. It is shown that the amorphous film exhibits $\mathrm{Cu}^{-}$ rie-Weiss-type paramagnetism with the effective magnetic moment of $4.2 \mu_{B} / \mathrm{Mn}$ ion and a small ferromagnetic contribution governed by the formation of a quasi-two-dimensional crystalline interfacial inclusions. The crystalline film with nanocrystalline randomly-oriented inclusions demonstrates a superposition of ferromagnetic (in the crystalline matrix) and superparamagnetic (in the inclusions) nature. The fitted average size of the superparamagnetic particles in the case of a Langevin function is coincident with that of the nanocrystalline clusters reveated in high-resolution electron-microscopy images. An increase in the applied magnetic field leads to a reduction in the average magnetic moment of superparamagnetic particles, which is due to an enhancement of the ferromagnetic coupling between the individual randomly-oriented crystallites. The completely crystalline film undergoes only a ferromagnetic transition with a saturation magnetization at $5 \mathrm{~K}$ of $2.73 \mu_{B} /$ Mn ion.
\end{abstract}

PACS: 71.30.+h, 75.47.Gk, 75.47.Lx

\section{Introduction}

The hole-doped perovskite manganites of the general formula $\mathrm{R}_{1-x} \mathrm{~A}_{x} \mathrm{MnO}_{3}$ ( $\mathrm{R}$ - rare-earth cation, A - alkali or alkaline-earth cation) have attracted considerable attention not only because of their interesting fundamental science, connected with the discovery of colossal magnetoresistance (CMR), but also their potential for device applications $[1,2]$. It was found recently that the lattice strain (and stress), accumulated owing to the epitaxial growth of a film, plays an important role in the formation of the spinand charge-ordered states in the CMR films [3-6]. The influence of the kind of single-crystalline substrates on the magnetic and electronic properties of manganite films has been quite will investigated $[7,8]$.
It was shown that the presence of grain boundaries in the polycrystalline manganites leads to a large MR effect over a wide temperature range below the Curie temperature $\left(T_{C}\right)$, whereas the CMR of the single-crystalline materials is restricted to a narrower temperature range just around $T_{C}$ [9-11]. On the other hand, the influence of structural quench disorder on the magnetic ordering is still poorly understood.

In this paper we report the peculiar results for amorphous (LCM1), a strain-free crystalline (with nanocrystalline mosaic-like inclusions) (LCM2), and lattice-strained crystalline (LCM3) $\mathrm{La}_{0.7} \mathrm{Ca}_{0.3} \mathrm{MnO}_{3}$ film. It was shown that the state of Mn spins can be controlled by a microstructure. We present an unusual type of the randomly-oriented nanocrystalline mosaic 
structure which manifests the magnetic-field-effected superparamagnetic behavior and sheds new light on the nature of magnetic ordering in the CMR materials.

\section{Experimental techniques}

All the films were prepared by rf-magnetron sputtering using a so-called «soft» (or powder) target [12]. The total pressure in chamber was $5 \cdot 10^{-2}$ Torr with a gas mixture of $\mathrm{Ar}$ and $\mathrm{O}_{2}(3: 1)$. The substrate was a $\mathrm{LaAlO}_{3}(001)$ single crystal (LAO) with a lattice parameter $a \simeq 0.379 \mathrm{~nm}$ for the pseudocubic symmetry. The substrate temperature during deposition was 20 (LCM1), 400 (LCM2), and $720{ }^{\circ} \mathrm{C}$ (LCM3). All the films have a thickness of $d \simeq 30 \mathrm{~nm}$. The $\theta-2 \theta$ $\mathrm{x}$-ray diffraction (XRD) patterns were obtained using a Rigaku diffractometer with $\mathrm{Cu} K_{\alpha}$ radiation. The lattice parameters evaluated directly from the XRD data were plotted against $\cos ^{2} \theta / \sin \theta$. With an extrapolated straight line to $\cos ^{2} \theta / \sin \theta=0$, a more precise determination of the lattice parameter was obtained. Cross-section specimens were prepared by the standard techniques using mechanical polishing followed by ion-beam milling under grazing incidence. The high-resolution electron-microscopy (HREM) studies were carried out using a Philips CM300UT-FEG microscope with a field emission gun operated at $300 \mathrm{kV}$. The point resolution of the microscope was of the order of $0.12 \mathrm{~nm}$. The resistance measurements were performed by using the four-probe method in a temperature range of 4.2-300 $\mathrm{K}$ and in a magnetic field up to $5 \mathrm{~T}$. The in-plane field-cooled (FC) and zero-field-cooled (ZFC) magnetization was taken with a Quantum Design SQUID magnetometer in a temperature range of $4.2-300 \mathrm{~K}$.

\section{Microstructure of the films}

Figures $1, a$ and $1, b$ present the (001) and the (002) Bragg reflections, respectively, for the LCM1 (1), LCM2 (2), and LCM3 (3) films and for the LAO substrate. The LCM1 film displays only a smooth plateau instead of a real Bragg peak. Therefore, the film deposited at a low temperature is in an amorphous phase. The LCM2 film shows weak Bragg peaks on a background of the same smooth plateau. The out-of-plane lattice parameter for the LCM2 film, $c \simeq 0.3866 \mathrm{~nm}$, is very close to that for the bulk $[13,14]$. This suggests that the incipient crystalline phase grows in equilibrium conditions without a significant influence of the substrate and is formed through the amorphous state. The LCM3 film, deposited at a high temperature, displays intense Bragg peaks with a larger out-of-plane lattice parameter, $c \simeq 0.3927 \mathrm{~nm}$, that is typical for the thin CMR films and explained by the accumula-

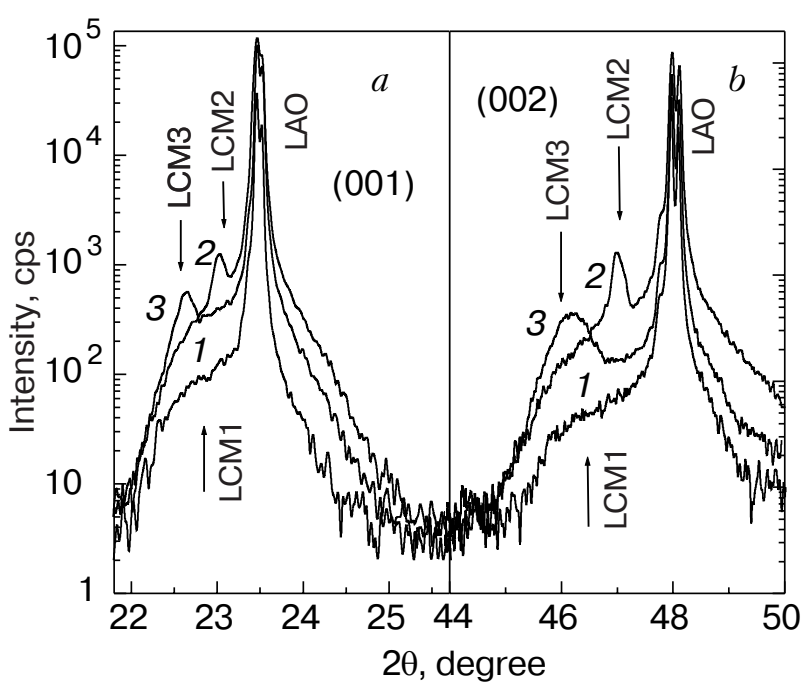

Fig.1. (001) (a) and (002) (b) XRD peaks for the LCM1 (1), LCM2 (2), and LCM3 (3) films. LAO denotes the substrate.

tion of an in-plane biaxial compressive strain during the deposition $[7,8,12,15]$.

Figure 2, $a$ is the high-magnification cross-sectional HREM image for the amorphous LCM1 film. It was obtained with an incident beam parallel to a cubic direction of the substrate and to the film/substrate interface. The inset in Fig. 2, $a$ displays the fast Fourier transform (FFT) of the HREM image across the interface. It is seen that the FFT produces a bright uniform halo instead of a rectangular pattern of circular spots. Only the slightly luminescent spots corresponding to the crystal lattice of the substrate are barely noticeable. This coincides with the XRD data and confirms an amorphous structure of the LCM1 film. On the other hand, Fig. $2, b$ shows that a trace of a crystalline phase is found on the substrate surface (denoted by white arrows). The analysis of the HREM images reveals that this crystalline phase does not cover the whole substrate surface and does not exceed one or two unit cells in thickness. Figure 3, $a$ demonstrates the same HREM image for the LCM2 film. Even though the FFT of LCM2 results in the formation of a rectangular pattern of spots (see inset A in Fig. 3,a), they are significantly smeared and include the bright halo that testifies for presence of the amorphous (or crystal-disordered) phase in the film. This sort of the off-structural inclusion is represented in Fig. 3, $b$ as area A and the FFT of its as the inset. The wide ring in FTT stands for the inclusions consists of the randomly-oriented nano-scale crystallites. Therefore, in this case we deal with the nanocrystalline mosaic microstructure rather than with an amorphous one, which was observed in LCM1. However, inset B in Fig. 3, $a$ shows that the matrix of LCM2 shows a per- 


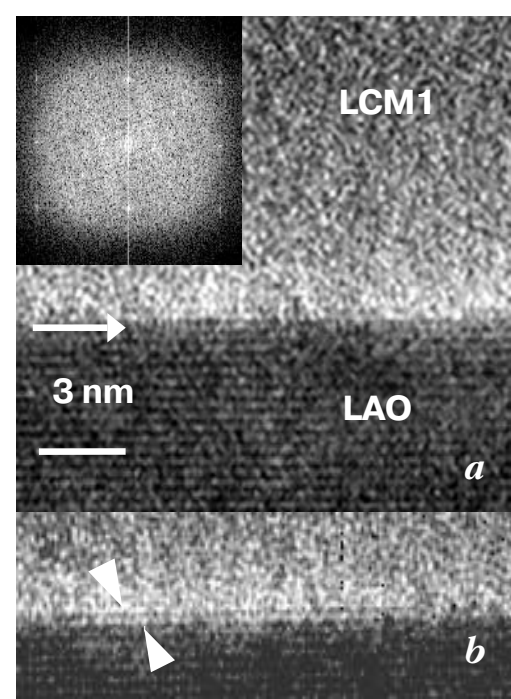

Fig. 2. Cross-sectional HREM image of the LCM1 film near the interface. The inset is the corresponding FFT $(a)$. The same image with a crystalline monolayer on the substrate surface, denoted by the white arrows $(b)$.

fect cubic crystalline lattice with the equal in-plane and out-of-plane lattice parameters, $a \simeq c \simeq 0.387 \mathrm{~nm}$, and right angle between the atom rows. It is matched with the XRD data and proofs a strainless crystal lattice of the LCM2 film. Figure 4 presents the HREM image for the

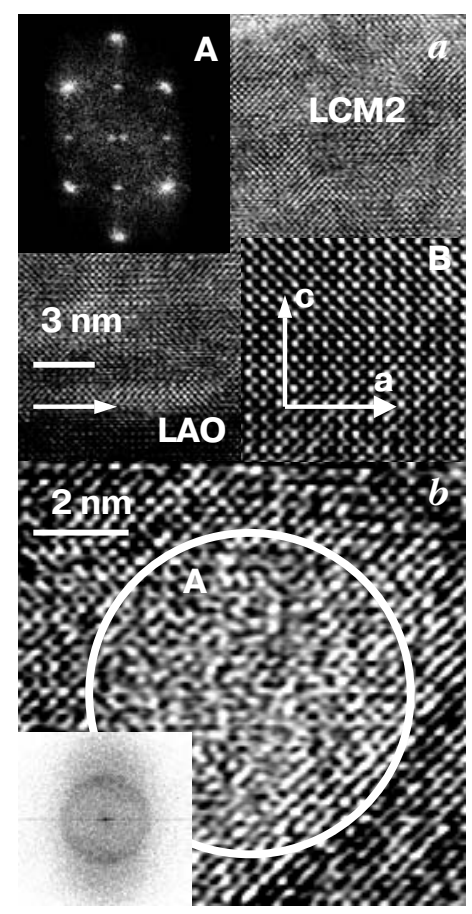

Fig. 3. Cross-sectional HREM image of the LCM2 film near the interface. Inset $\mathrm{A}$ is the corresponding FFT. Inset $\mathrm{B}$ represents the atomic lattice of the crystalline matrix (a). The HREM image with an off-structural inclusion designated by area A. Inset is the FFT of area A (b).

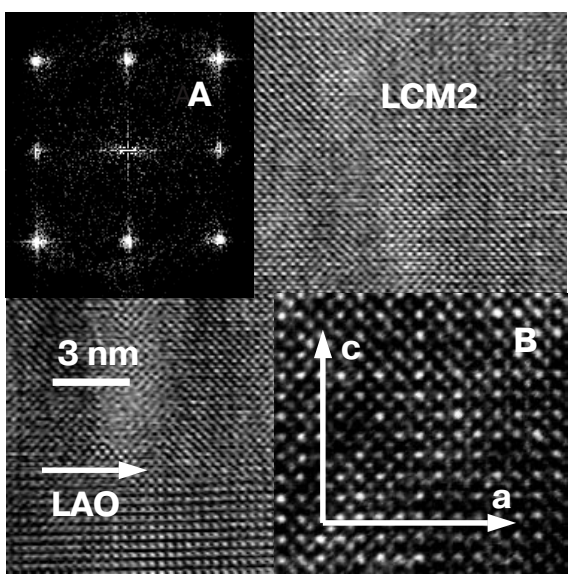

Fig. 4. Cross-sectional HREM image of the LCM3 film near the interface. Inset $\mathrm{A}$ is the corresponding FFT. Inset $B$ represents the atomic lattice of the film.

LCM3 film. In this case the FFT produces a well-defined rectangular pattern of circular spots (see inset A) which manifests formation of the perfect crystal lattice. However, together with the perpendicular crystal axes, a tetragonal distortion of the lattice $(c / a \simeq 1.026)$ is also found in inset B. The estimated in-plane lattice parameter for the LCM3 film, $a \simeq 0.3826 \mathrm{~nm}$, is smaller than that for the bulk compound, $a \simeq 0.3858 \mathrm{~nm}$ [14]. This difference leads to formation of the aforementioned in-plane biaxial compressive strain, $\varepsilon_{100}=\left(a_{\text {film }}-a_{\text {bulk }}\right) / a_{\text {bulk }}$, and an out-of-plane uniaxial tensile strain, $\varepsilon_{001}==\left(c_{\text {film }}-c_{\text {bulk }}\right) / c_{\text {bulk }}$. The performed calculations show that, in this case, $\varepsilon \simeq$ $\simeq-0.83$ and $\varepsilon \simeq 1.79 \%$. As far as the microstructural measurements are summarized, it is noted that LCM1 is mainly in an amorphous crystal structure, LCM2 contains a complex of the strainless crystalline matrix with nanocrystalline mosaic-type inclusions, and finally LCM3 represents a strained crystal lattice with a slight tetragonal distortion.

\section{Experimental results}

Figure 5 shows both FC (solid) and ZFC (open) temperature-dependent magnetization curves, $M(T)$, at a magnetic field $H=100$ Oe for the LCM1 $(a)$, the LCM2 (b) and the LCM3 (c) films. The amorphous LCM1 film demonstrates an $M(T)$ behavior which is typical for paramagnetic (PM) materials [16]. At the same time, two peculiarities, a minor splitting between the ZFC and FC $M(T)$ curves and a slight decrease in the magnetization near the expected Curie point (denoted by arrow in Fig. 5,a), indicate the presence of a ferromagnetic (FM) phase. It is claimed that a small FM contribution is connected with the mentioned ultrathin crystalline layer on the substrate surface (see Fig. 2,b). The inset in Fig. 5, $a$ displays the $M(H)$ dependence at room temperature, which 


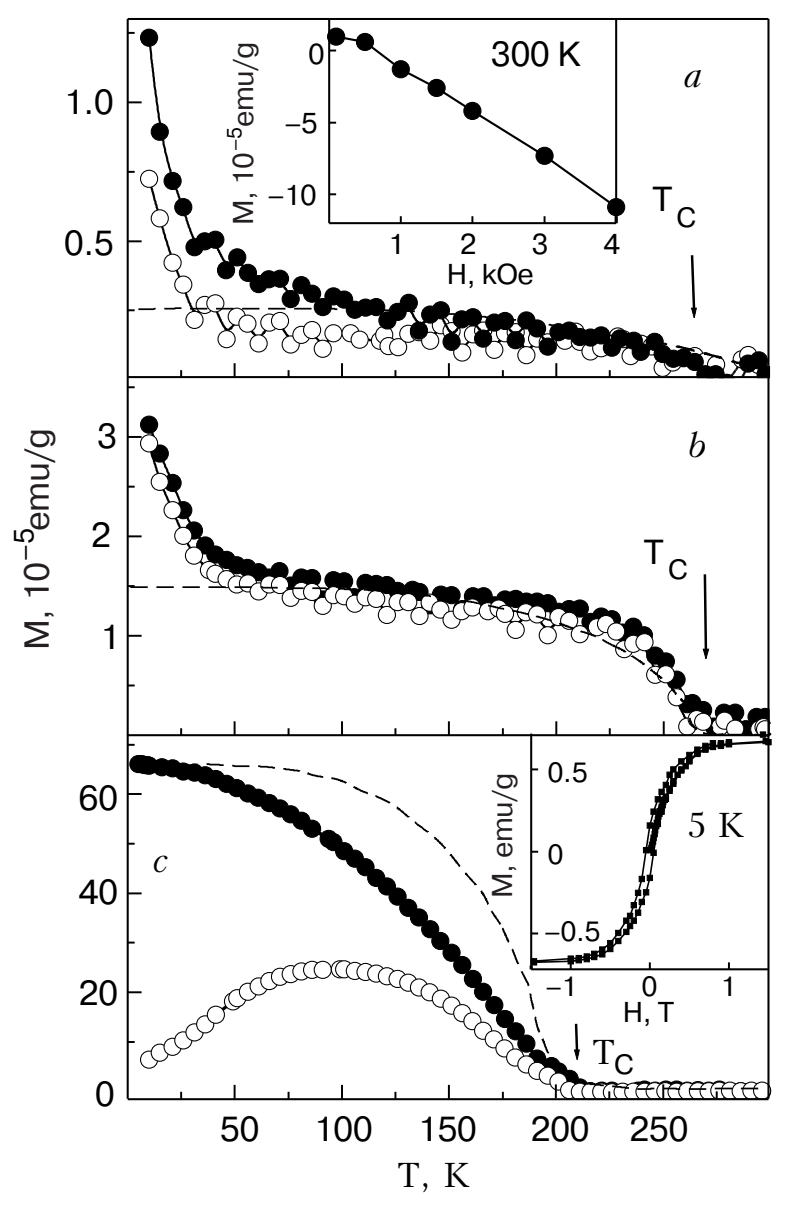

Fig. 5. Field-cooled (solid) and zero-field-cooled (open) magnetization curves for the LCM1 $(a)$, LCM2 (b), and LCM3 (c) films. The inset in ( $a$ ) displays the field-dependent magnetization at $300 \mathrm{~K}$ for LCM1. The inset in (c) shows the hysteresis loop at $5 \mathrm{~K}$ for LCM3. The dashed lines are the theoretical curves, based on the mean-field approximation for ferromagnetic materials. The lines are guides to the eye.

demonstrates a diamagnetic behavior, introduced by LAO. The measurements performed reveal that the diamagnetic contribution is about $-3.3 \cdot 10^{-3} \mathrm{emu} / \mathrm{g}$ for all the prepared films. This contribution was first subtracted from the magnetization curves for the analyses. Figure $5, b$ shows that the $M(T)$ dependence for the LCM2 film represents a superposition of two contributions: the well-defined FM transition at $T_{C} \simeq 260 \mathrm{~K}$, and the paramagnetic term which is manifested by a rapid increase of the $M(T)$ at $T \rightarrow 0$. Taking into account the XRD and HREM data, one can conclude that the FM phase belongs to the crystalline matrix and the PM phase corresponds to the nanocrystalline inclusions. Figure 5,c shows that the crystalline LCM3 film undergoes only a FM transition at $T_{C} \simeq 204 \mathrm{~K}$, which is lower than that for LCM2 and can be explained by the generated lattice strains in the film [17]. The inset in Fig. 5,c presents the hysteresis loop of the magnetization, measured at $5 \mathrm{~K}$, for the LCM3 film. It is seen that the magnetization is saturated at $H_{s} \simeq 9000 \mathrm{Oe}$ and that the coercive field is about 500 Oe. The carried out analysis shows that the saturation magnetization does not exceed of 2.73 $\mu_{B} / \mathrm{Mn}$ that is typical for the lattice strained thin films [16] and is much smaller than observed for the bulk, $3.5 \mu_{B} /$ Mn [17].

Figure 6 displays the temperature dependence of the resistance, $R(T)$, for the LCM3 film with (solid) and without (open) an applied magnetic field of $5 \mathrm{~T}$. Unfortunately, our setting was limited to $10^{7} \Omega$, and the $R(T)$ could not be measured for LCM1 and LCM2, since their resistances are about $10^{12}$ and $10^{7} \Omega$ at room temperature, respectively. The magnetic field was directed parallel to the film surface and at a right angle to the transport current. The metal-insulator (MI) transition is observed at $T_{P} \simeq 180 \mathrm{~K}$ and is much lower than $T_{C}$. Inset A in Fig. 6 shows the temperature dependence of the MR for the same film. The MR value is defined by $100 \%[R(0)-R(H)] / R(H)$, where $R(0)$ and $R(H)$ are the resistances without and with a magnetic field, respectively. It is seen that the $\mathrm{MR}(T)$ dependence has a peak-like shape and reaches almost $1500 \%$ at the maximum.

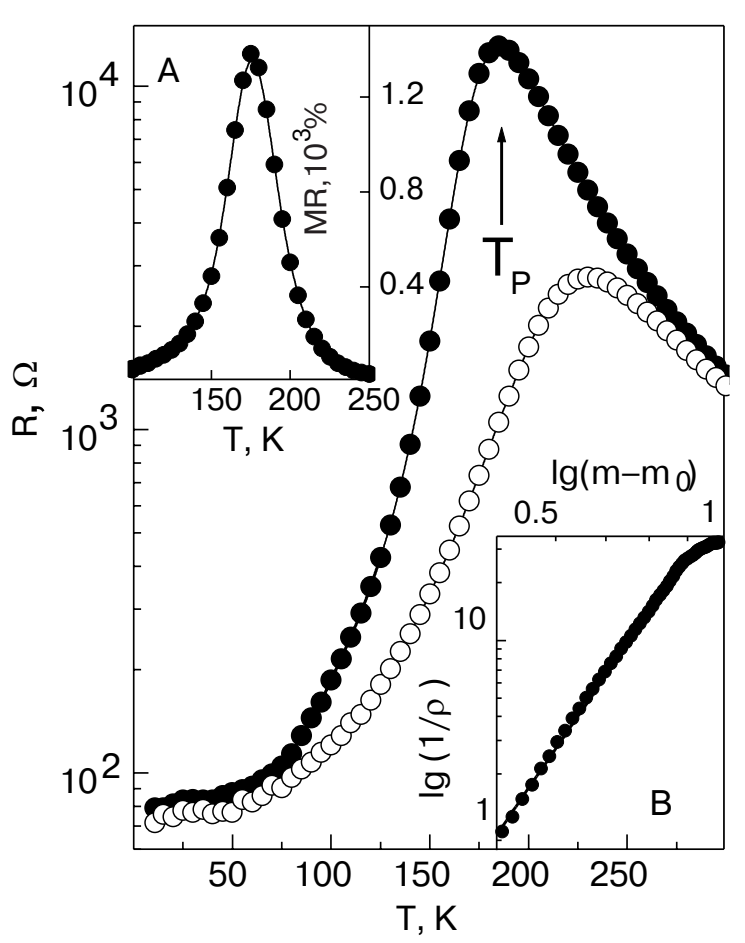

Fig. 6. Temperature dependence of the resistance for LCM3 film without (open) and with (solid) an applied magnetic field of $5 \mathrm{~T}$. The lines are guides to the eye. Inset $\mathrm{A}$ shows the temperature dependence of the MR ratio in an applied magnetic field of $5 \mathrm{~T}$. Inset $\mathrm{B}$ displays the $\lg (1 / \rho)$ versus $\lg \left(m-m_{0}\right)$ plot with the slope corresponding to the percolation exponent $t \simeq 5.7$. 


\section{Discussion}

As above-mentioned, the amorphous LCM1 film demonstrates mainly paramagnetic $M(T)$ behavior with a trace of FM. Thus, the expression for the total magnetization of LCM1 can be written as $M(T, H)=$ $=M^{P M}(T, H)+M^{F M}(T, H)$. The paramagnetic contribution to the magnetization could be written, in the whole temperature range, as [16]

$$
M^{P M}(T, H)=\left(\chi_{0}+\frac{C_{C W}}{T+\theta}\right) H,
$$

where $\chi_{0}$ is the temperature-independent susceptibility, and the second term is a Curie-Weiss (CW)-type susceptibility with a constant $C_{C W}$ and a characteristic temperature $\theta$. Figure $7, a$ demonstrates that the experimental data for LCM1 is excellently described by the $\mathrm{CW}$ expression with the following fitting parameters: $\chi_{0} \simeq 2.5 \cdot 10^{-4} \mathrm{~cm}^{3} / \mathrm{g}, C_{C W} \simeq 1.04 \cdot 10^{-2} \mathrm{~cm}^{3} / \mathrm{g}$, and $\theta=3 \mathrm{~K}$. The estimated from $C_{C W}$ effective moment
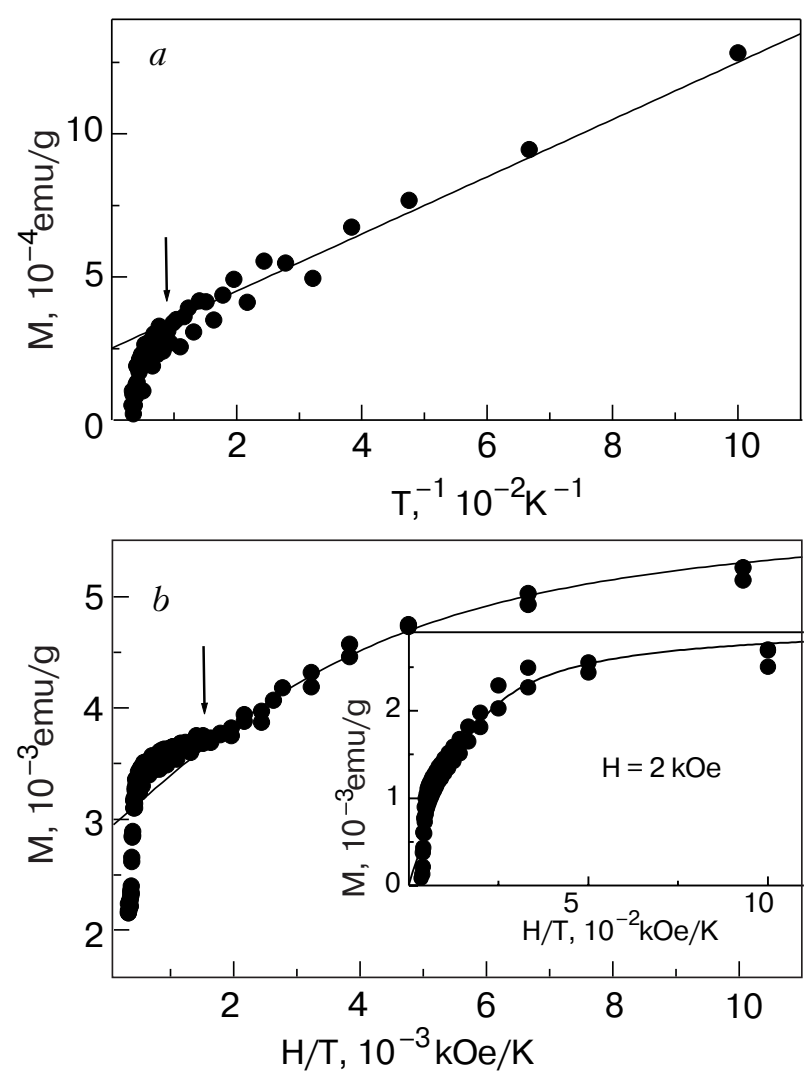

Fig. 7. $M$ versus $T^{-1}$ plot for the LCM1 film at $H=$ $=100$ Oe. The solid line represents the CW-type paramagnetic approximation. The arrow indicates the deviation of the experimental curve from the CW-type straight line, reflecting the presence of a FM phase $(a)$. $M$ versus $H / T$ plot for the LCM2 film at $H=100$ Oe. The solid line represents a Langevin function describing the magnetic behavior of superparamagnetic particles. The arrow indicates the presence of a FM phase. The inset is the same plot at $H=2 \mathrm{kOe}(b)$. turn out to be $\mu_{\text {eff }} \simeq 4.2 \mu_{B} / \mathrm{Mn}$, that is almost coincident with the theoretical value, $\mu_{\text {eff }}^{\text {theo }} \simeq 4.6 \mu_{B} / \mathrm{Mn}$, which is obtained from the following expression:

$$
\mu_{\mathrm{eff}}^{\text {theo }}=g \sqrt{x S_{1}\left(S_{1}+1\right)+(1-x) S_{2}\left(S_{2}+1\right)} .
$$

Here $x$ is the Ca concentration, $S_{1}=3 / 2$ and $S_{2}=2$ are the spin values of $\mathrm{Mn}^{4+}$ and $\mathrm{Mn}^{3+}$ ions, respectively, and $g=2$ is the Lande factor. Therefore, one can conclude that the amorphous LCM1 film is mainly a typical $\mathrm{CW}$-type paramagnet. On the other hand, the magnetization decreases sharply and deviates from a straight line in the range of small values for $T^{-1}(T \geq 130-150 \mathrm{~K})$, as denoted by an arrow in Fig. 7,a. It is reasonable to suggest that the observed deviation from the CW law is governed by the FM transition of the crystalline interfacial layers. The mean-field theory gives the following temperature dependence of the spontaneous magnetization for a ferromagnet below $T_{C}[16]$ :

$$
\frac{M^{F M}}{M_{0}^{F M}}=\tanh \left[\frac{M^{F M}}{M_{0}^{F M}} \cdot \frac{T_{C}}{T}+\frac{\chi_{\mathrm{diff}} H}{M_{0}^{F M} T}\right],
$$

where $M_{0}^{F M}$ is the spontaneous magnetization at $T \rightarrow 0$ and $\chi_{\text {diff }}$ is the differential susceptibility. The dashed lines in Figs. 5, $a, b$, and 5, $c$ are the theoretical curves calculated in the mean-field approximation. $\chi_{\text {diff }}=2 \cdot 19 \cdot 10^{-4} \mathrm{~cm}^{3} / \mathrm{g}$ was taken from the magnetization hysteresis loop for LCM3 (see inset in Fig. 5,c,) which is already in the FM state at a magnetic field of 100 Oe.

Figure $7, b$ shows the $M(T, H)$ versus $H / T$ plot for the LCM2 film, measured at $H=100 \mathrm{Oe}$, and the inset presents the same plot at $H=2 \mathrm{kOe}$. It is seen that the experimental curves cannot be described by the $\mathrm{CW}$ approximation, since they do not demonstrate a linear behavior in the whole temperature range. Such a nonlinearity of the $M(H / T)$ is more typical for superparamagnetic (SPM) particles and can be described by the Langevin function [16]:

$$
M^{S P M}(T, H)=M_{S}^{S P M}\left[\operatorname{coth}\left(\frac{\mu H}{k_{B} T}\right)-\frac{k_{B} T}{\mu H}\right],
$$

where $M_{S}^{S P M}$ is the saturation magnetization of the SPM phase and $\mu$ is the average magnetic moment of the SPM particles. The solid lines represent the Langevin functions fitted closest to the experimental data. These lines correspond to an average magnetic moment of the SPM particles of $3000 \mu_{B}$ at $H=100$ Oe and $800 \mu_{B}$ at $H=2 \mathrm{kOe}$. By taking $3.5 \mu_{B} / \mathrm{Mn}$ atom [18] and assuming a spherical shape of the SPM clusters with a volume of $\pi D^{3} / 6$, where $D$ is the average diameter, we estimate their average diameter to 
be $D \simeq 5 \mathrm{~nm}$ for 100 Oe and $\simeq 3 \mathrm{~nm}$ for $2 \mathrm{kOe}$. Analysis of the HREM images (see Fig. 3,b) shows that the randomly-oriented crystallites in inclusions have a similar size to this estimation for the SPM clusters. Therefore, one can conclude that nanocrystalline clusters play the role of the SPM particles in this film. The observed decrease in the average moment of the SPM particles in an applied magnetic field can be explained by a partial SPM to FM transition due to an enhancement of the ferromagnetic coupling between adjacent nanocrystalline clusters. It is provided by a high conductive transparency of boundaries in the such type of a nanocrystalline mosaic structure. In this case the arrangement of magnetic moments in the increasing field can lead to a recovery of the double exchange interaction between neighboring crystallites.

As already described, the observed decrease in $T_{C}$ for the LCM3 film is explained by an influence of the lattice strain which was accumulated in the film during the epitaxial growth. For weaker strains and a cubic symmetry, the Curie point can be expressed, according to Millis model, by [17]

$$
T_{C}(\epsilon)=T_{C}(\epsilon=0)\left(1-\alpha \epsilon_{B}-\frac{1}{2} \Delta \epsilon_{J T}^{2}\right),
$$

where $\epsilon_{B}=\left(2 \epsilon_{100}+\epsilon_{001}\right)$ is the bulk strain, $\epsilon_{J T}=$ $=\sqrt{2 / 3}\left(\epsilon_{001}-\epsilon_{100}\right)$ is the Jahn-Teller strain, $\alpha=$ $=\left(1 / T_{C}\right)\left(d T_{C} / d \epsilon_{B}\right)$, and $\Delta=\left(1 / T_{C}\right)\left(d^{2} T_{C} / d \varepsilon_{J T}^{2}\right)$. The magnitudes of $\alpha$ and $\Delta$ represent the relative weights for symmetry-conserving bulk and symmetry-breaking Jahn-Teller strains, respectively. According to the theoretical model, [17] $\alpha \simeq 10$ for a reasonable electron-phonon coupling $(0.5 \leq \lambda \leq 1)$ in these compounds. Taking into account that the Curie temperature for the strain-free bulk $\mathrm{La}_{0.7} \mathrm{Ca}_{0.3} \mathrm{MnO}_{3}$ compound is $T_{C}(\varepsilon=0) \simeq 265 \mathrm{~K}[14,19]$ and using the obtained values of $\varepsilon_{100}$ and $\varepsilon_{001}$ and $T_{C}$ for LCM3, we determined $\Delta$ to be about 950 , which is of the same order of magnitude as the prediction of the theoretical model [17]. A possible explanation for this discrepancy is that the lattice mismatch between LCM3 and LAO is so large that the effect of structural disorder becomes dominant over the strain effect and that the role of Jahn-Teller distortion in formation of the ferromagnetic ordering is distinctly decreased. On the other hand, the additional deformation of the crystal lattice can be connected with the so-called «chemical-pressure» effect which is provided by the high diffusion rate of oxygen in these materials. Therefore, the modified lattice parameters are caused by both the simple elastic strain and the nonstoichiometry. In any case, the obtained results reflect a strong correlation between crystal lattice distortion and the electronic and magnetic states in CMR materials.

According to percolation theory, the conductivity can expressed as $\sigma \sim\left(p-p_{0}\right)^{t}$, where $p$ is the concentration of a metallic phase and $p_{0}$ is its critical value [20]. Taking into account that the value of the spontaneous magnetization is proportional to the concentration of the FM metallic phase and $p_{0}=0.395$ for three-dimensional systems [21], we can express the percolating conductivity for LCM3 by the formula $(1 / \rho) \sim\left(m-m_{0}\right)^{t}$, where $\rho=R(T) / R\left(T_{P}\right), R\left(T_{P}\right)$ is the resistance at temperature of the MI transition, $m=M(T) / M(0)$, and $m_{0}=0.395$. Inset $\mathrm{B}$ in Fig. 6 exhibits a linear dependence of $\lg (1 / \rho)$ versus $\lg \left(m-m_{0}\right)$, and the slope of the curve corresponds to the exponent $t \simeq 5.7$. This experimental value for $t$ is very close to that $(t \simeq 5.3$ ) obtained by a numerical calculation for the three-dimensional system, considering the spin effects [21]. Therefore, one can conclude that the MI transition in the LCM3 film has a percolating nature. It is reasonable to suggest that the nonuniformly distributed lattice strains in the film play a key role in blocking the complete transition to the FM phase within a narrow temperature range.

\section{Conclusion}

In summary, $\mathrm{La}_{0.7} \mathrm{Ca}_{0.3} \mathrm{MnO}_{3}$ films were successfully prepared to have three characteristic structures: amorphous, a strain-free crystalline (with randomly-oriented nanocrystalline mosaic inclusions) and lattice-strained crystalline, and their the magnetic properties were investigated. It was shown that the amorphous film exhibits a CW-type PM behavior with a freely moving of the individual $\mathrm{Mn}$ spins and a small FM contribution governed by the quasi-two-dimensional crystalline interfacial phase. The crystalline film with nanocrystalline mosaic inclusions demonstrates a superposition of the FM (corresponding to the crystalline matrix) and the SPM (to the nanocrystalline mosaic inclusions) contributions. The fitted average size of SPM particles using Langevin function is coincident with that of the nanocrystalline mosaic clusters represented by the HREM images. An increase in the applied magnetic field leads to a reduction in the average magnetic moment of SPM particles, which is due to an enhancement of the FM coupling between the individual crystallites in a mosaic inclusions and a partial SPM to FM transition. The observed suppression of contribution from the Jahn-Teller distortion to the FM ordering, with increasing the lattice mismatch between substrate and lattice-strained film, manifests a physical limit of the small-strain approach [17] in describing the magnetic properties of manganite films. 
This work was supported by the KOSEF through the Quantum Photonic Science Research Center.

1. Y. Tokura and Y. Tomioka, J. Magn. Magn. Mater. 200, 1 (1999).

2. A.P. Raminez, J. Phys. C9, 8171 (1997).

3. F. Tsui, M.C. Smoak, T.K. Nath, and C.B. Eom, Appl. Phys. Lett. 76, 2421 (2000).

4. R.A. Rao, D. Lavric, T.K. Nath, C.B. Eom, L. Wu, and F. Tsui, Appl. Phys. Lett. 73, 3294 (1998).

5. S. Jacob, T. Roch, F.S. Razavi, G.M. Gross, and H.-U. Habermeier, J. Appl. Phys. 91, 2232 (2002).

6. A. Biswas, M. Rajeswari, R.C. Srivastava, T. Venkatesan, R.L. Green, Q. Lu, A.L. de Lozanne, and A.J. Millis, Phys. Rev. B63, 184424 (2001).

7. O.I. Lebedev, G. Van Tendeloo, S. Amelinckx, H.L. Ju, and K.M. Krishnan, Philos. Mag. 80, 673 (2000).

8. J.R. Sun, C.F. Yeung, K. Zhou, L.Z. Zhou, C.H. Leung, H.K. Wong, and B.G. Shen, Appl. Phys. Lett. 76, 1164 (2000).

9. H.Y. Hwang, S.-W. Cheong, N.P. Ong, and B. Batlogg, Phys. Rev. Lett. 77, 2041 (1996).

10. A. Gupta, G.Q. Gong, G. Xiao, P.R. Duncombe, P. Lecoeur, P. Trouilloud, Y.Y. Wang, V.P. Dravid, and J.Z. Sun, Phys. Rev. B54, R15 629 (1996).

11. R. Gross, L. Alff, B. Büchner, B.H. Freitag, C. Höfener, J. Klein, Y. Lu, W. Mader, J.B. Philipp, M.S.R. Rao, P. Reutler, S. Ritter, S. Thienhaus, S. Uhlenbruck, and B. Wiedenhorst, J. Magn. Magn. Mater. 211, 150 (2000).
12. V.G. Prokhorov, G.G. Kaminsky, V.A. Komashko, J.S. Park, and Y.P. Lee, J. Appl. Phys. 90, 1055 (2001).

13. R. Mahendiran, S.K. Tiwary, A.K. Raychaudhuri, and T.V. Ramakrishnan, Phys. Rev. B53, 3348 (1996).

14. Y.H. Li, K.A. Thomas, P.S.I.P.N. de Silva, L.F. Cohen, A. Goyal, M. Rajeswari, N.D. Mathur, M.G. Blamire, J.E. Evetts, T. Venkatesan, and J.L. MacManus-Driscoll, J. Mater. Res. 13, 2161 (1998).

15. V.G. Prokhorov, Y.P. Lee, V.A. Komashko, V.L. Svetchnikov, and J.S. Park, Phys. Status Solidi A196, 74 (2003).

16. B.D. Cullity, Introduction to Magnetic Materials, Addison-Wesley, New York (1872).

17. A.J. Millis, T. Darling, and A. Migliori, J. Appl. Phys. 83, 1588 (1998).

18. E.O. Wollan and W.C. Koehler, Phys. Rev. 100, 545 (1955).

19. G.L. Liu, J.S. Zhou, and J.B. Goodenough, Phys. Rev. B64, 144414 (2001).

20. E. Dagotto, T. Hotta, and A. Moreo, Phys. Rep. 344, 1 (2001).

21. Y. Xiong, S.-Q. Shen, and X.C. Xie, Phys. Rev. B63, 140418 (2001). 